Conclusions Prior to a dedicated hepatologist the vast majority of those presenting with AVB have a history of previous AVB and are potentially avoidable. With the advent of dedicated banding lists (as well as closer follow up with more robust secondary prophylaxis) there has been a major reduction of AVB presenting. There has been a shift of 'new' AVB unknown to the system rather than previous existing patients having undergone prior therapy. This has equated to a significant reduction in AVB of $30 \%$ during period $1 \%$ and $56 \%$ during period 2. Dedicated 'surveillance' lists such as for Barrett's have shown to reduce the incidence of late presentation of disease and we propose that dedicated varices surveillance and banding lists can reduce acute admissions.

\section{PTH-089 HEPATOCELLULAR CARCINOMA (HCC) SURVEILLANCE IN CLINICAL PRACTICE: A DISTRICT GENERAL HOSPITAL SINGLE-CENTRE EXPERIENCE}

Selena Dixon*, Amy Longhurst, Jack Murgatroyd, Sulleman Moreea. Bradford Royal Infirmary, Bradford, UK

\subsection{6/gutjnl-2018-BSGAbstracts.245}

Introduction Hepatocellular carcinoma (HCC) mortality and incidence is increasing worldwide. Current guidelines recommend biannual surveillance with ultrasound (USS) and/or alpha-fetoprotein (AFP) to ensure early detection and prompt treatment, yet the benefit on patient outcomes is uncertain in the absence of high quality data. We aimed to describe the merits of HCC surveillance in a single-centre cohort with an ethnically diverse population.

Methods We retrospectively identified patients diagnosed with HCC from 2010 to 2017. We determined whether HCC occurred on surveillance or not. We collected information including demographic data, aetiology and severity of liver disease, AFP levels, tumour size, initial treatment, survival status and cause of death.

Results 101 cases were identified. Median age was 71 years (range 47-94), 75\% were male. 63\% were white and 25\% from South Asian background. The commonest aetiology was Non-Alcoholic Fatty Liver Disease (NAFLD, 22.8\%), followed by Alcohol-Related Liver Disease (ARLD, 19.8\%), Hepatitis C (HCV, 21.8\%) and Hepatitis B (HBV, 5\%). 7/22 patients with HCV had achieved SVR. Only 1 received direct-acting antivirals (DAA) prior to HCC diagnosis.

25/101 patients were diagnosed on HCC surveillance; 11/ 101 presented with acute decompensated cirrhosis ( 9 were under a surveillance programme, 2 had failed to attend); 43/ 101 presented with symptoms and 22/101 were incidental findings. HCV was the predominant aetiology in those presenting symptomatically.

AFP was normal in half of all cases. Of those on surveillance, $63 \%$ had AFP measured prior to diagnosis and $8.5 \%$ had a raised AFP when initial imaging was normal. 57\% patients were Child's A, 38\% Child's B and 5\% Child's C at diagnosis.

Patients were more likely to have HCC diagnosed at an early stage on surveillance $(68.6 \%$ vs $30.3 \%)$ and receive curative treatment $(22.8 \%$ vs $12.1 \%)$ than the non-surveillance group. 1 and 3 year survival rates were greater on surveillance (67.7\% vs $41.1 \%$ and $22.2 \%$ vs $8.16 \%$, respectively). Median survival after diagnosis in the surveillance group was greater than those presenting for the first time.
Conclusions Surveillance was associated with earlier stage cancers and receipt of potentially curative treatment. However, patients known to secondary care made up a minority of HCC diagnoses. Improving identification and diagnosis of cirrhosis in primary care may therefore help identify at-risk patients earlier, although not all patients will engage with follow-up.

AFP measurement may identify additional cases of HCC that go undetected by USS, but should be weighed against potential patient harms from false-positive Results. Further studies should continue to inform an optimum HCC surveillance strategy.

\section{PTH-090 SURVIVAL AFTER A DIAGNOSIS OF HEPATOCELLULAR CARCINOMA}

Robert Driver*, David Chizhande, Rebecca Jones, lan Rowe. Leeds Teaching Hospitals, Leeds, UK

\subsection{6/gutjnl-2018-BSGAbstracts.246}

Introduction Survival estimates for different Barcelona Clinic Liver Cancer (BCLC) stages in hepatocellular carcinoma (HCC) contained in the EASL-EORTC Clinical Practice Guidelines rely on outcomes from randomised control trials and meta-analysis of pooled data. To identify areas for development to facilitate improvements in outcomes we aimed to provide an insight into HCC survival outcomes outside a clinical trials setting by presenting a large experience of patients referred with HCC to a regional hepatobiliary cancer centre in the UK.

Methods All patients referred to the Hepatobiliary Cancer Multidisciplinary Team with a diagnosis of HCC over a two year period (January 2013 to December 2014) were included. Patients were stratified by their initial treatment modality according to the BCLC classification. Kaplan-Meier survival analysis was used to compare outcomes by initial treatment allocation.

Results Among 356 patients (median age 66 years, 291 (82\%) male), the most frequent underlying disease aetiologies were hepatitis $\mathrm{C}$ and alcohol-related liver disease. Overall survival at 3 years after diagnosis was $38 \%$ and 146 patients (41\%) received treatment with curative intent. The 3 year survival for liver transplant was $84 \%$ (56 patients) and for resection it was $89 \%$ (46 patients). The median survival for radiofrequency ablation was 45 months (44 patients) and for transarterial chemoembolization (TACE) it was 18 months (72 patients). For patients receiving sorafenib as first-line therapy, the median survival was 9.6 months (12 patients) and for those receiving best supportive care (BSC) it was 3.4 months (126 patients).

Conclusions These estimates of overall survival are consistent with those published in the EASL-EORTC Clinical Practice Guidelines and demonstrate that these figures give a reliable estimate of overall survival in a real-world experience. Over one third of patients were unsuitable for anti-cancer therapy at presentation and only a minority received treatment with curative intent. This highlights areas for potential improvement in outcomes particularly through early diagnosis of cirrhosis, facilitating treatment of the underlying cause of liver disease as well as the implementation of surveillance for HCC. Screening strategies for cirrhosis should be investigated to determine whether these can reduce overall mortality, including that from HCC. 